\title{
Identification of boosepivirus B in U.S. calves
}

\author{
Ben M. Hause ${ }^{1}\left[\right.$ [ Eric Nelson ${ }^{1}$ Jane Christopher-Hennings ${ }^{1}$
}

Received: 3 June 2021 / Accepted: 19 July 2021 / Published online: 15 September 2021

(c) The Author(s), under exclusive licence to Springer-Verlag GmbH Austria, part of Springer Nature 2021

\begin{abstract}
Bovine enteric disease has a complex etiology that can include viral, bacterial, and parasitic pathogens and is a significant source of losses due to morbidity and mortality. Boosepivirus was identified in calves with enteric disease with unclear etiology in Japan in 2009 and has not been reported elsewhere. Metagenomic sequencing and PCR here identified boosepivirus in bovine enteric disease diagnostic submissions from six states in the USA with 98\% sequence identity to members of the species Boosepivirus B. In all cases, boosepivirus was identified as a coinfection with the established pathogens bovine coronavirus, bovine rotavirus, and cryptosporidia. Further research is needed to determine the clinical significance of boosepivirus infection.
\end{abstract}

Enteric disease is a significant health concern for bovine calves and a leading cause of morbidity and mortality early in life [1]. Clinical disease is often multifactorial, with viral, bacterial, and parasitic pathogens interacting with host and environmental factors. Bovine coronavirus (BCV) and bovine rotavirus (BRV) are etiologic agents of enteric disease; however, a multitude of viruses from diverse families have been identified in clinical specimens with unresolved clinical significance [2, 3]. For the family Picornaviridae, members of the genera Enterovirus, Kobuvirus, Hunnivirus, and Bopivirus, have been identified in cattle [4-9]. More recently, bovine picornavirus, reclassified as boosepivirus (BPV), was identified in fecal samples from calves with enteric disease in Japan in 2009 [10]. While two bovine species of BPV have been recognized, a member of a third species, Boosepivirus $C$, was identified in sheep (unpublished). To our knowledge, BPV has not been reported apart from its initial characterization in Japan in 2009.

Handling Editor: Ana Cristina Bratanich.

The genome sequence of boosepivirus B strain 21-0305 was deposited in the GenBank database under accession no. MZ052226.

Ben M. Hause

benjamin.hause@sdstate.edu

1 Animal Disease Research and Diagnostic Laboratory, Department of Veterinary and Biomedical Sciences, South Dakota State University, Brookings, SD 57007, USA
Pooled intestinal tissue and fecal swabs were collected from 10-day-old calves in Missouri, USA, exhibiting prolonged diarrhea lasting for more than five days, resulting in death. Metagenomic sequencing was performed to identify possible pathogens as described previously [11]. A sample was clarified by centrifugation and digested with a nuclease cocktail, followed by reverse transcription and secondstrand synthesis with barcoded primers containing a 3' random hexamer. Following purification, DNA was amplified using barcode-specific primers, followed by construction of a Nextera XT sequencing library. Sequencing was performed using a MiSeq sequencer with paired 151-base-pair chemistry. Reads trimmed of adapter sequences were assembled $d e$ novo using CLC Genomics software with contigs identified by BLASTx using BLAST2Go.

One of the 45 contigs assembled from the approximately 4.5 million reads was identified as BPV. The contig was 7,365 nucleotides (nt) in length and was assembled from 8,257 reads $(0.18 \%)$. As open reading frame (ORF) analysis indicated that the 3' end of the single large ORF was incomplete, rapid amplification of cDNA ends was performed using a Roche 5'/3'-RACE Kit, $2^{\text {nd }}$ generation, using primers designed to anneal near the 3' end of the genome, bovpic6615: 5'-GTCGCCACAGGTTCTGCTGTTG and bovpic6863: 5'-GGGTATGCCATCTGGAACTAGC. The remainder of the genome was amplified by amplification of overlapping approximately 1,000-base-pair (bp) segments. The final genome sequence was confirmed by Sanger sequencing. The sequence for BPV 21-0305 was submitted 
to the GenBank database under the accession number MZ052226.

The BPV genome sequence is $7,440 \mathrm{nt}$ in length and is $86 \%$ identical to those of BPV isolates recovered from Japan in 2009, according to BLASTn analysis. The genome contains a single large ORF, encoding a protein of 2,341 amino acids that is $98 \%$ identical to that of BPV by BLASTp analysis. The 5' untranslated region, consisting of 314 nucleotides, was incomplete based on the length of 553 nucleotides reported for BPV.

A second contig 8,421 nt in length was also identified as a complete bovine kobuvirus (BKV) genome. This contig was comprised of 373,465 reads $(8.3 \%)$. The BKV genome was $94 \%$ identical to that of bovine kobuvirus strain IL35164 (GenBank accession no. MN336260), which was identified in calves with diarrhea in the USA. [12]. The predicted 2,463-amino-acid protein encoded by the open reading frame was $98.2 \%$ identical to that of MN336260. No other mammalian viruses were identified.

To investigate the evolutionary history of BPV 21-0305, the region of the genome encoding the $3 \mathrm{D}$ protein was identified by alignment with reference sequences. Reference sequences for picornavirus 3D nucleotides were downloaded from the Picornaviridae resources website from the International Committee of Taxonomy of Viruses [13]. Sequences were aligned by ClustalW as implemented in MEGA X. Phylogenetic analysis was next performed by the maximumlikelihood method using the best-fitting GTR+G+I model of evolution. Tree topology was evaluated using 1,000 bootstrap replicates. Boosepivirus strain 21-0305 formed a sister clade to one formed by multiple strains of BPV recently reclassified as members of the species Boosepivirus $B$ (Fig. 1). This, along with the $98 \%$ identity of the polyprotein of strain 21-0305 to members of Boosepivirus B, suggests that strain 21-0305 belongs to the species Boosepivirus $B$ [4]. Together, the boosepiviruses formed a well-supported monophyletic clade, supporting their designation as members of a separate genus. Apart from other boosepiviruses, strain 21-0305 was similar to unclassified canine and bat picornaviruses.

A TaqMan assay was designed targeting the $3 \mathrm{D}$ region of BPV, using the primers Forw, 5'-TTTCCATTCCTC ATACACCCTG, and Rev, 5'-TCTCTTCCGTTGTGCCAT $\mathrm{G}$, and the probe 5'-FAM-ATTCCTCGACCAGCGAAT GCTCTC. Next, RNA was isolated from a collection of 48 bovine enteric disease diagnostic submissions submitted for diagnostic testing. Real-time reverse transcription PCR was performed using TaqMan Fast Virus 1-Step Master Mix according to the manufacturer's instructions. Six of the $48(12.5 \%)$ samples were positive for BPV, including the original sample in which the virus was detected. Cycle threshold values ranged from 18.1 to 34.2 (Table 1). The six positive samples originated from diseased calves from four Midwestern states as well as California. The 12.5\% prevalence, combined with detection in multiple states across a broad geographic area, suggests that BPV infections are common in U.S. cattle.

As enteric disease is often multifactorial, we explored coinfections of BPV with significant enteric disease pathogens in the six BPV-positive samples. Bovine coronavirus and BRV were detected by RT-PCR, while cryptosporidia were detected microscopically (Table 1). Enteric pathogenic bacteria were identified by aerobic culture. All BPV-positive cases were also positive for at least one etiologic pathogen of enteric disease. These results suggest that BPV is commonly found in coinfections with known enteric pathogens. Interestingly, for cases 1 and 4, the $\mathrm{Ct}$ value for $\mathrm{BPV}$ was considerably lower than for $\mathrm{BCV}$ and $\mathrm{BRV}$, suggesting that BPV was present in the clinical samples at higher titers than BCV and BRV.

Routine metagenomic sequencing of a diagnostic submission here identified BPV for the first time since its original and only description from Japan in 2009. While BPV was originally identified in clinical samples from calves with enteric disease that tested negative in routine tests for BCV, BRV, and bovine torovirus, here, BPV was always found in association with BCV and BRV infections. Interestingly, the initial description of BPV noted that, for three of the five samples positive for BPV, BKV was also present. We also identified BPV and BKV coinfection. Bovine kobuvirus has been detected in calves with enteric disease in many countries. In China, BKV was significantly associated with calf diarrhea [14]. In the USA, BKV infection was associated with histologic lesions consistent with enteric disease [12]. In the BPV-positive sample here, BKV accounted for $8.3 \%$ of the total reads, while only $0.18 \%$ of the reads mapped to BPV, suggesting that BKV was present at much higher levels in our enteric disease sample. Further research is needed to determine the clinical significance of boosepivirus infection. 
Fig. 1 Phylogenetic analysis of the genome region encoding the 3D protein of bovine picornavirus. Sequences were aligned using ClustalW, and a phylogenetic tree was constructed by the maximum-likelihood method using the GTR $+\mathrm{G}+\mathrm{I}$ model of evolution with 1,000 bootstrap replicates. Bootstrap values 0.70 or greater are shown

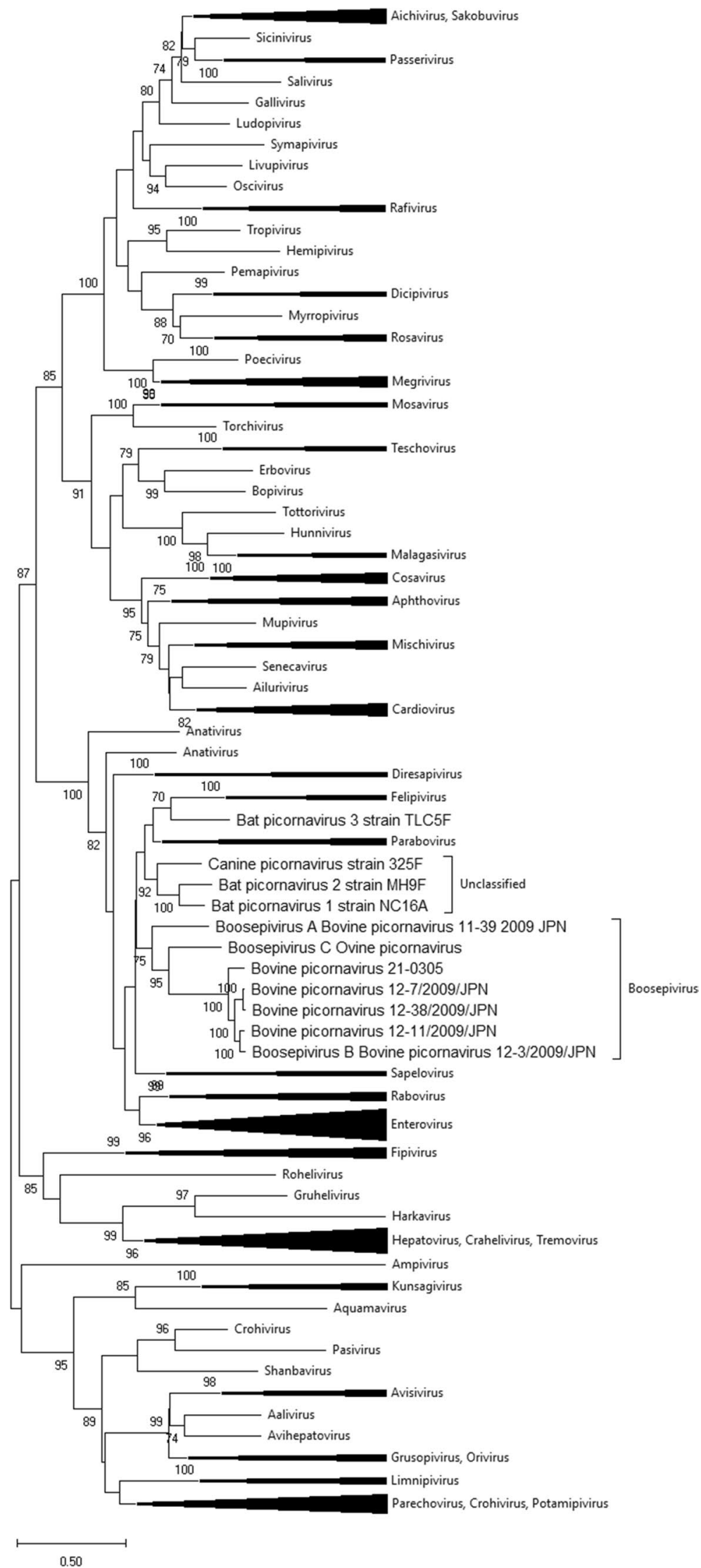


Table 1 Bovine enteric disease diagnostic test results for boosepivirus (BPV)-positive diagnostic submissions

\begin{tabular}{lllllll}
\hline Case & State & BPV & BRV & BCV & Cryptosporidium & Aerobic culture \\
\hline 1 & South Dakota & 20.9 & 35.7 & 29.8 & Negative & Negative \\
2 & Nebraska & 28.6 & 18.2 & 31.8 & Positive & Negative \\
3 & South Dakota & 21.5 & 14.5 & Negative & Positive & Negative \\
$4^{*}$ & Missouri & 18.1 & 27 & Negative & NT & Negative \\
5 & California & 28.9 & 29.4 & 36.1 & NT & NT \\
6 & Minnesota & 34.2 & Negative & 25.2 & Positive & Negative \\
\hline
\end{tabular}

Bovine rotavirus (BRV) and bovine coronavirus (BCV) were detected by quantitative RT-PCR, and the cycle threshold value for positive samples is shown. The presence (positive) or absence (negative) of cryptosporidium is indicated as well as significant organisms detected by aerobic culture

$N T$ not tested

*Original case where BPV was detected by metagenomic sequencing
Acknowledgements We would like to thank Cambridge Technologies for assistance with sample procurement. This work was supported in part by the United States Department of Agriculture and South Dakota Agricultural Experiment Station Animal Health and Hatch funds.

Author contributions $\mathrm{BH}$ : methodology, analysis, writing. EN and $\mathrm{JH}$ : funding acquisition, resources.

Funding No specific funding supported this research. Unspecified funds from the United States Department of Agriculture and South Dakota Agricultural Experiment Station were used to conduct this research in addition to user fees for diagnostic testing.

Availability of data and material The genome sequence for boosepivirus B strain 21-0305 was submitted to GenBank under accession no. MZ052226.

\section{Declarations}

Conflict of interest The authors declare that there are no conflicts of interest.

Ethics statement The animal specimens used in this study were sourced from samples submitted for diagnostic testing by licensed veterinarians as part of their professional responsibilities. As such, no specific institutional animal care and use committee approval was required.

\section{References}

1. Gomez DE, Weese JS (2017) Viral enteritis in calves. Can Vet J58:1267-1274

2. Brunauer M, Roch FF, Conrady B (2021) Prevalence of worldwide neonatal calf diarrhea caused by bovine rotavirus in combination with bovine coronavirus, Escherichia coli $\mathrm{K} 99$ and Cryptosporidium spp.: a meta-analysis. Animals 11:1014. https://doi.org/10. 3390/ani11041014

3. Vlasova AN, Saif LJ (2021) Bovine coronavirus and associated diseases. Front Vet Sci 8:643220. https://doi.org/10.3389/fvets. 2021.643220
4. Zell R, Delwart E, Gorbalenya AE, Hovi T, King AMQ, Knowles NJ, Lindberg AM, Pallansch MA, Palmenberg AC, Reuter G, Simmonds P, Skern T, Stanway G, Yamashita T, ICTV Report Consortium (1997) ICTV virus taxonomy profile: Picornaviridae. J Gen Virol 98:2421-2422. https://doi.org/10.1099/jgv.0.000911

5. Tsuchiaka S, Rahpaya S, Otomaru K, Aoki H, Kishimoto M, Naoi Y, Omatsu T, Sano K, Okazaki-Terashima S, Katayama Y, Oba M, Nagai M, Mizutani T (2017) Identification of a novel bovine enterovirus possessing highly divergent amino acid sequencing in capsid protein. BMC Microbiol 17:18. https://doi.org/10.1186/ s12866-016-0923-0

6. Zhu L, Xing Z, Gai X, Li S, San Z, Wang X (2014) Identification of a novel enterovirus $\mathrm{E}$ isolates HY12 from cattle with severe respiratory and enteric diseases. PLoS ONE 9:e97730. https:// doi.org/10.1371/journal.pone.0097730

7. Reuter G, Egyed L (2009) Bovine Kobuvirus in Europe. Emerg Infect Dis 15:822-823. https://doi.org/10.3201/eid1505.081427

8. Isidan H, Turan T, Atasoy MO, Sozdutmaz I, Irehan B (2019) Detection and first molecular characterization of three picornaviruses from diarrhoeic calves in Turkey. Acta Vet Hung 67:463476. https://doi.org/10.1556/004.2019.046

9. Laszlo Z, Pankovics P, Reuter G, Csagola A, Balint A, Albert M, Boros A (2021) Multiple types of novel enteric bopiviruses (Picornaviridae) with the possibility of interspecies transmission identified from cloven-hoofed domestic livestock (ovine, caprine, and bovine) in Hungary. Viruses 13:66. https://doi.org/10.3390/ v13010066

10. Nagai M, Omatsu T, Aoki H, Kaku Y, Belsham GJ, Haga K, Naoi Y, Sano K, Umetsu M, Shiokawa M, Tsuchiaka S, Furuya T, Okazaki S, Katayama Y, Oba M, Shirai J, Katayama K, Mizutani T (2015) Identification and complete genome analysis of a novel bovine picornavirus in Japan. Virus Res 210:205-212. https://doi. org/10.1016/j.virusres.2015.08.001

11. Hause BM, Collin EA, Anderson J, Hesse RA, Anderson G (2015) Bovine rhinitis viruses are common in cattle with bovine respiratory disease. PLoS ONE 10:e0121998. https://doi.org/10.1371/ journal.pone.0121998

12. Wang L, Fredrickson R, Duncan M, Samuelson J, Hsiao SH (2020) Bovine kobuvirus in calves with diarrhea, United States. Emerg Infect Dis 26:176-178. https://doi.org/10.3201/eid2601. 191227

13. Resources: Picornaviridae-Picornaviridae-Positive-sense RNA Viruses-ICTV. http://ictvonline.org. Accessed 27 May 2021

14. Li H, Tang C, Yue H (2019) Molecular detection and genomic characteristics of bovine kobuvirus from dairy calves in China. Infect Genet Evol 74:103939. https://doi.org/10.1016/j.meegid. 2019.103939 
Publisher's Note Springer Nature remains neutral with regard to jurisdictional claims in published maps and institutional affiliations. 